\title{
Control of real-time MRI with a 3D controller during radiofrequency ablation
}

\section{Vanessa Zurawka, Rüdiger Hoffmann, Oliver Burgert}

Vanessa Zurawka, Rüdiger Hoffmann, Oliver Burgert, "Control of real-time MRI with a 3D controller during radiofrequency ablation," Proc. SPIE 10576, Medical Imaging 2018: Image-Guided Procedures, Robotic Interventions, and Modeling, 1057621 (23 March 2018); doi: 10.1117/12.2293705

SPIE. Event: SPIE Medical Imaging, 2018, Houston, Texas, United States 


\title{
Control of real-time MRI with a 3D-controller during radiofrequency ablation
}

\author{
Vanessa Zurawka ${ }^{1}$, Rüdiger Hoffmann ${ }^{2}$, Oliver Burgert ${ }^{1}$ \\ ${ }^{1}$ Reutlingen University, 72762 Reutlingen, Germany \\ ${ }^{2}$ Diagnostische und Interventionelle Radiologie, Universitätsklinikum Tübingen, Germany
}

\begin{abstract}
Radiofrequency ablation is an ablation technique to treat tumors with focused heat. Computer tomography, ultrasound and magnetic resonance imaging (MRI) are imaging modalities which can be used for image-guided procedures. MRI offers several advantages in comparison to the other imaging modalities, such as radiation-free fluoroscopic imaging, temperature mapping, a high-soft-tissue contrast and free selection of imaging planes. This work addresses the application of 3Dcontrollers for controlling interventional, fluoroscopic MR sequences at the scenario of MR-guided radiofrequency ablation of hepatic malignancies. During this procedure, the interventionalist can monitor the targeting of the tumor with near-real time fluoroscopic sequences. In general, adjustments of the imaging planes are necessary during tumor targeting, which is performed by an assistant in the control room. Therefore, communication between the interventionalist in the scanner room and the assistant in the control room is essential. However, verbal communication is impaired due to the loud scanning noises. Alternatively, non-verbal communication between the two persons is possible, however limited to a few gestures and susceptible to misunderstandings. This work is analyzing different 3D-controllers to enable control of interventional MR sequences during MR-guided procedures directly by the interventionalist. Leap Motion, Wii Remote, SpaceNavigator, Phantom Omni and Foot Switch where selected. For that a simulation was built in C++ with VTK to feign the real scenario for test purposes. Previous results showed that Leap Motion is not suitable for the application while Wii Remote and Foot Switch are possible input devices. Final evaluation showed a generally time reduction with the use of 3D-controllers. Best results were reached with Wii Remote in 34 seconds. Handholding input devices like Wii Remote have further potential to integrate them in real environment to reduce intervention time.
\end{abstract}

Keywords: Image-guided therapy, radiofrequency ablation, MRI-guidance, 3D controller, human machine interface

\section{DESCRIPTION OF PURPOSE}

Radiofrequency ablation (RFA) is a medical procedure which aims on destroying tumors by applying heat locally. For this, an electrode is inserted in the tumor, which is heated by applying alternating currents in the radiofrequency range. For a successful treatment, the heat source must be carefully placed in the right position based on pre-interventional planning and medical experience. To ensure proper positioning of the electrode, imaging is used during the intervention. Direct image guidance can be performed based on ultrasound, computed tomography or magnetic resonance imaging (MRI). MRI has the advantages of a high-soft-tissue contrast, free selection of imaging planes and MR-fluoroscopic sequences with no radiation exposure [1]. A major disadvantage is the relative long duration of MR-guided procedures. For MR-fluoroscopic sequences, three image planes are acquired in near-real time, not the whole relevant volume [2]. Since the electrode (and maybe the patient) is moving during the intervention, the image planes have to be re-adjusted during the intervention. So far, the manufacturer of those systems offers interfaces for interventions. Further Pinkernelle et al. [3] made a study with a wireless PC Mouse to control some basic options of the MRI. While the interventionalist in the scanner room can monitor the tumor targeting on a screen, the assistant in the control room is navigating the software. Struggling with the loud gradient noises it would be useful that the interventionalist could control the real-time MRI himself, especially in the case of regaining the ablation needle while the targeting process. This would solve the communication problem and further save time. This work is analyzing several 3D-Controllers and implementing them in a simulation. For evaluation, interventionalists at University Hospital Tübingen are going to test the simulation to collect feedback.

Medical Imaging 2018: Image-Guided Procedures, Robotic Interventions, and Modeling, edited by

Baowei Fei, Robert J. Webster III, Proc. of SPIE Vol. 10576, 1057621 · @ 2018 SPIE

CCC code: $1605-7422 / 18 / \$ 18 \cdot$ doi: $10.1117 / 12.2293705$ 


\section{METHODS}

\subsection{Requirements Engineering}

For identifying the requirements, observation of interventions and surveys [4] with interventionalists and assistants where used. Both took place at University Hospital Tübingen.

Three observations were performed, each one immediately before, during and directly after intervention. Since all observations revealed similar processes, no further observations were done.

The main purpose of the survey was to discover problems in the current interaction scenario, to find out different opinions about the intended usage of a self-guided system, and general conditions of the intervention. Three interventionalists and two medical-technical assistants were interviewed.

The results are shown in table 1 and 2 in chapter 3.1.

\subsection{Simulation of the slice positioning process}

In order to be able to test different input devices in a repeatable scenario, a simulation system for the slice positioning and orientation process was built. Implementation was done in C++ using the Visualization Toolkit (VTK). We used a 3D dataset of a liver; the ablation needle was painted in several positions inside the volume to generate various exercises. The hole overview of the software components is shown in class diagram of simulation software in figure 1.

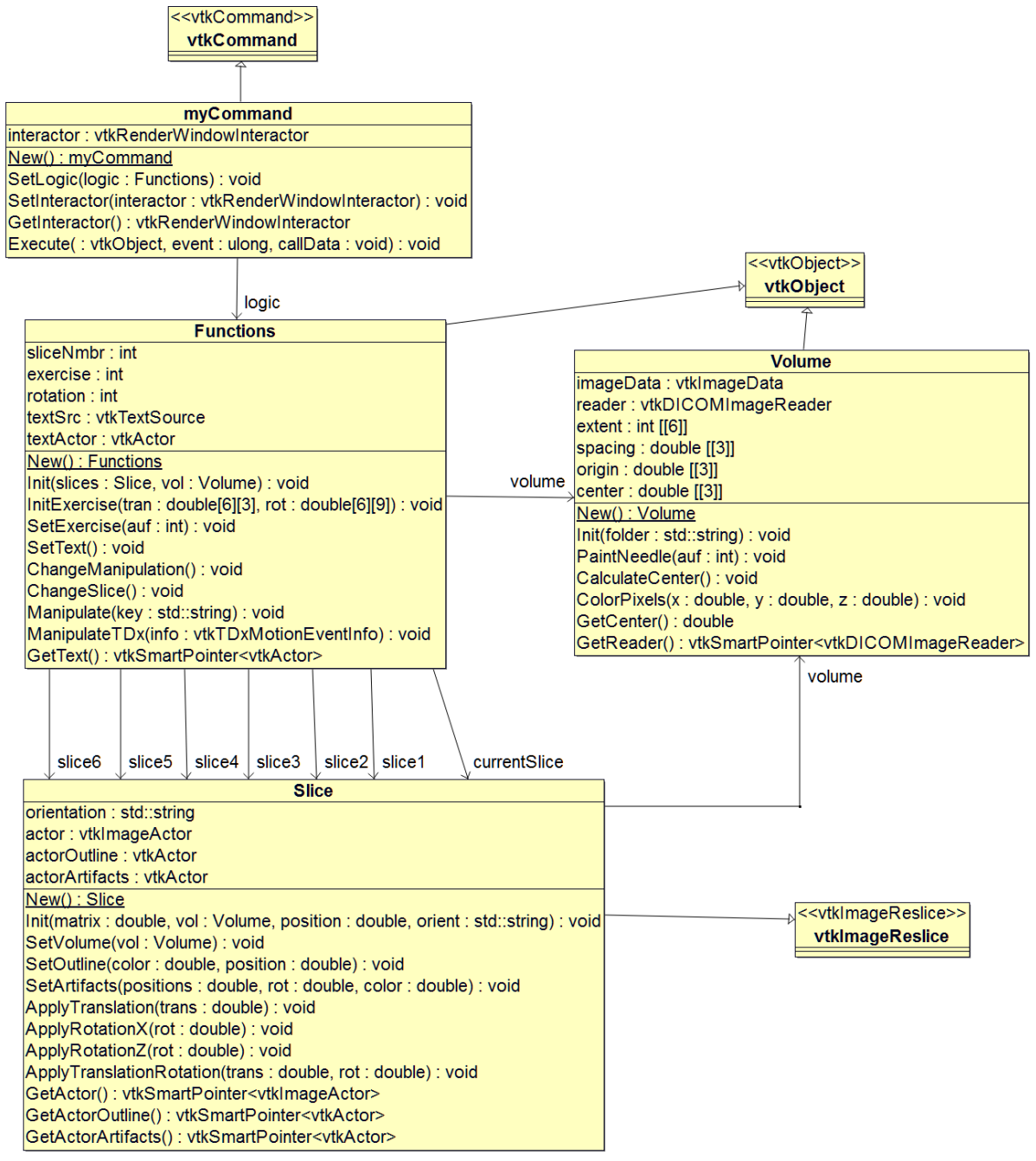

Figure 1. class diagram of simulation software 
Figure 2 shows the software interface. The needle is the black line and the colored lines are the resulting image artifacts of the other image planes. The top row represents the reference images, which would be the needle itself in the real intervention. The bottom row are the pictures the interventionalist would see on his screen during the intervention where he must re-position the slices in order to see the needle again in all imaging planes.

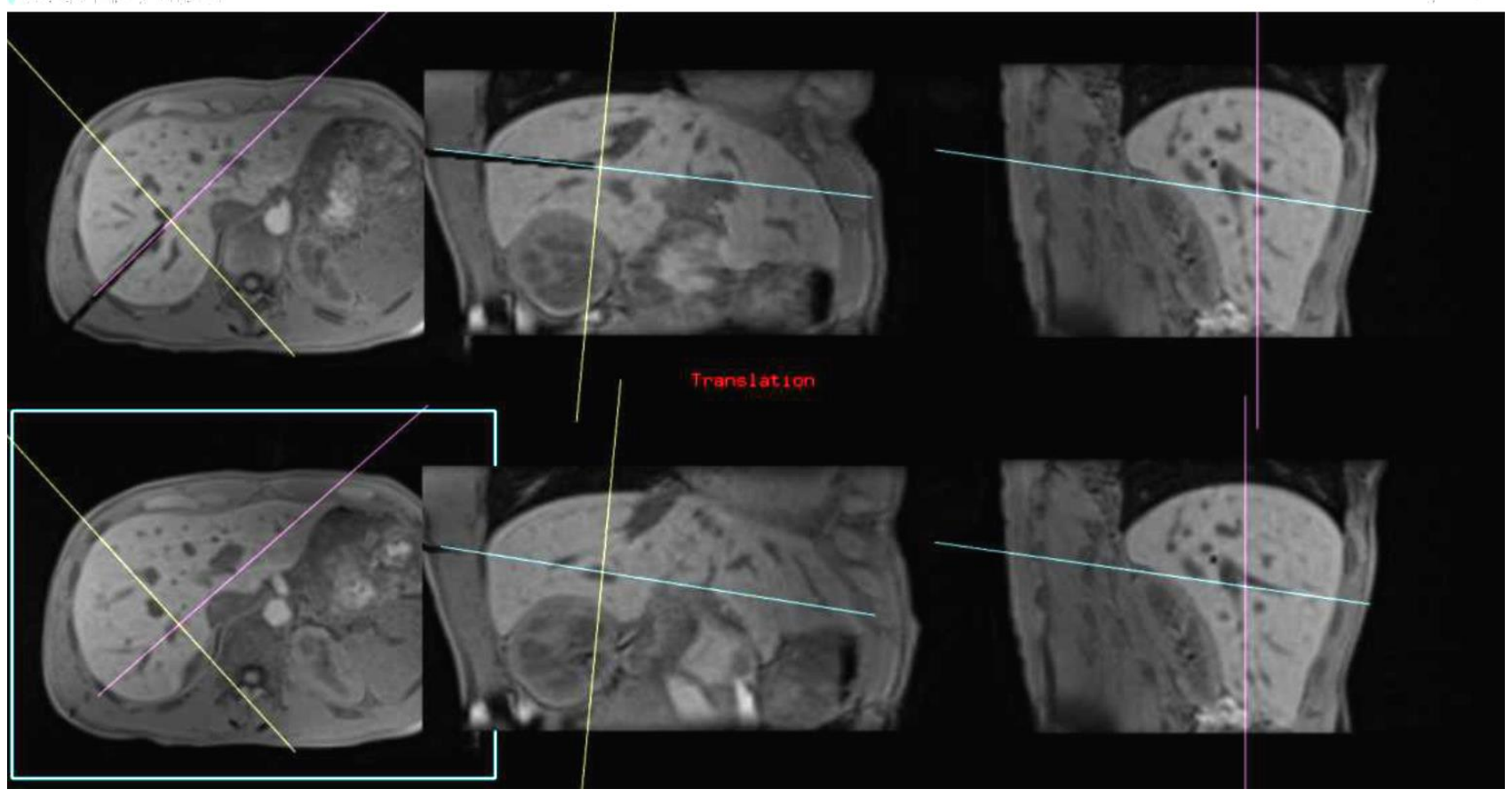

Figure 2. Simulation Software used for the evaluation of various input devices. Top row shows the three image planes with a virtual needle (black) inserted in the liver, the lower rows shows the same data set where the interventionalist has to re-position the slices to find the needle again.

The initialization process of an exercise is composed of different steps. The user needs to enter a number between 1 and 4 on his keyboard to start the associated exercise. The initialization starts with painting the needle in the 3D dataset in a certain position. Afterwards the correct translation and rotation are applied on the reference planes and the moved translation and rotation are applied on the simulated fluoroscopic image planes. This process is shown in figure 3.

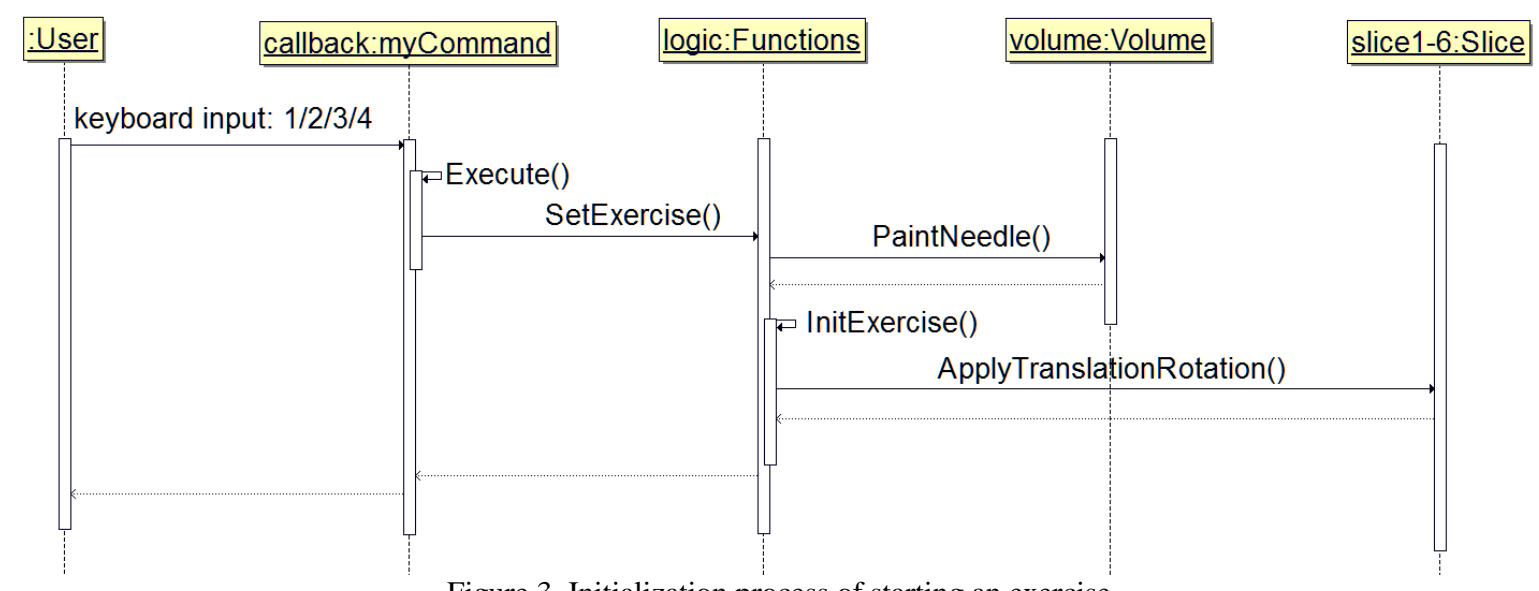

Figure 3. Initialization process of starting an exercise 
The user has different options of manipulating the simulated fluoroscopic image planes. One option is to translate on the coordinate axis forward and backward. The other option would be to rotate the image plane around two coordinate axes. For this option the user needs to change the manipulation mode to rotation with pressing the appropriate button. Then he has the option to rotate around two axes separately. On every input device the corresponding buttons are on various positions, but the software recognizes them as normal keyboard commands. This process is shown in figure 4.

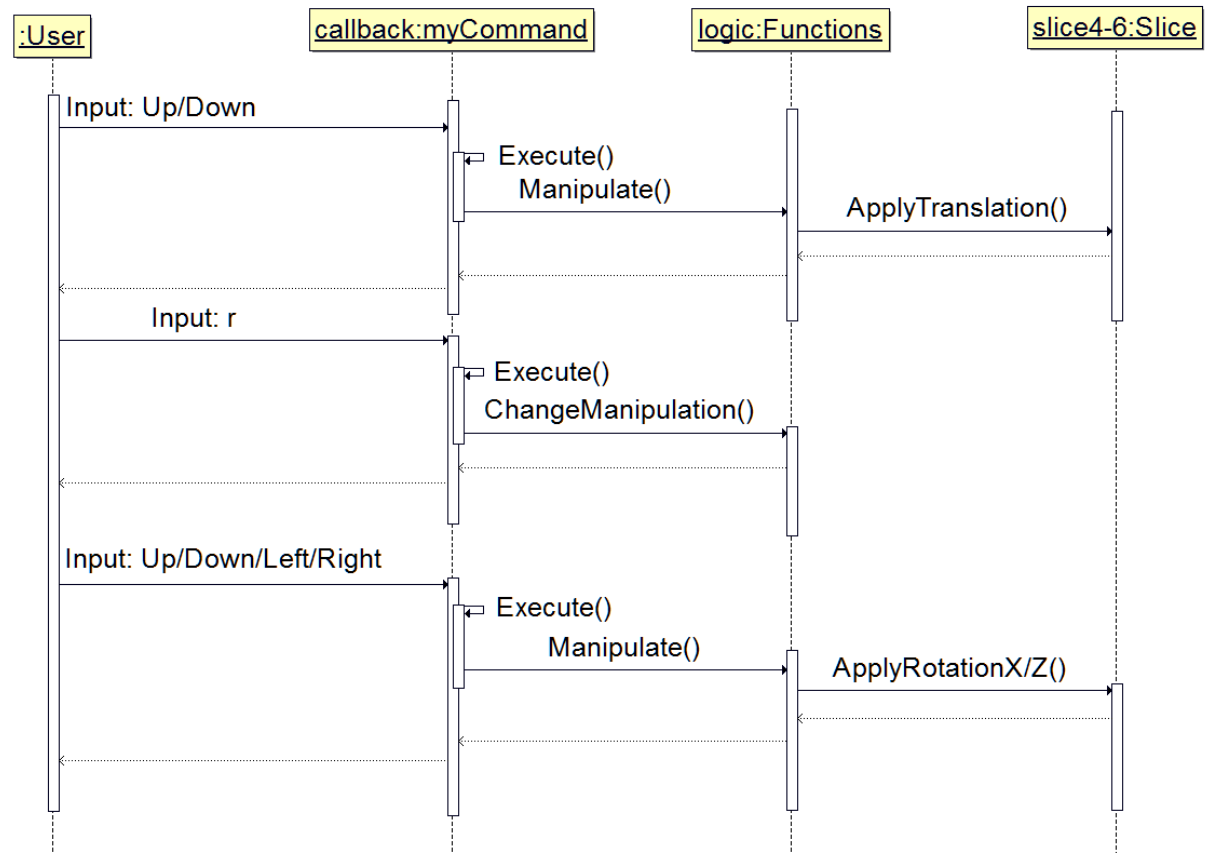

Figure 4. user input process to manipulate translation and rotation

\subsection{Evaluation}

Pre-evaluation took place before implementing the simulation. The purpose was to evaluate the general acceptance and handling of the following 3D-controllers: Leap Motion Controller, Phantom Omni, Wii Remote, Space Navigator and Foot Switch. The exercise for the pre-evaluation was to freely navigate through a $3 \mathrm{D}$ dataset to experience the $3 \mathrm{D}$-controllers and assess them afterwards.

Final evaluation will be performed at the University Hospital Tübingen in August 2017. The set-up will be as follows: Five interventionalists are evaluating each input device by using the simulator described in 2.2 . The procedure will be starting with a three minutes acclimation of the 3D-controller to get used to the handling. Afterwards they must solve five different tasks to regain the needle while time is measured. This will be repeated with all controllers. The controllers are randomly assigned to the evaluation sequence to avoid training effect biases. The measurements of time are set in comparison to the time measurements of the real scenario in intervention which is shown in Figure 5. Altogether the time consumption is 2 to 3 minutes in the real scenario to regain the needle. The goal of the simulation should be then to solve every exercise in less than that. 


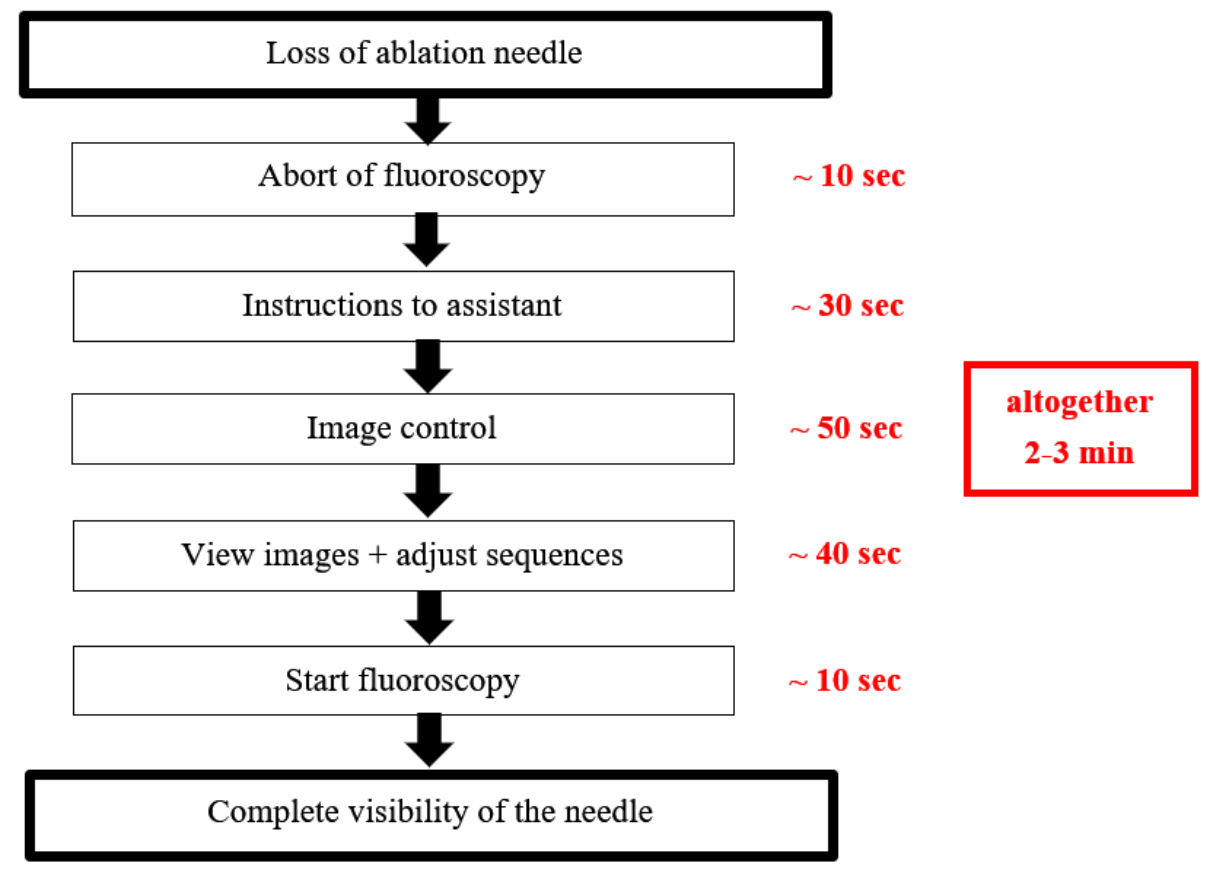

Figure 5. time measurements of the real scenario in intervention

For comparable results the evaluation process needs to offer certain steps. First the interventionalist needs time for acclimation because of the new interaction techniques and input devices where a reasonable time should be 3 minutes. We did not want to exceed a few minutes of acclimation because the devices should be easy to interact with and in everyday life there is no time for learning a new interaction technique. As a second point he needs to look at the task before solving it because in real scenario he would have placed the needle already and would know where the needle takes place. Therefor 3 seconds should be reasonable. After all the time measuring is starting and the interventionalist starts solving the task. The exercise is complete when the needle is fully visible in every image plane. After this the next input device takes place and the procedure is repeated. In the end the interventionalist is getting interviewed about his point of view how the interaction felt like and which device he can imagine working in real scenario. The hole process is shown in Figure 6 . The results of final evaluation are shown in table 3 in chapter 3.2.

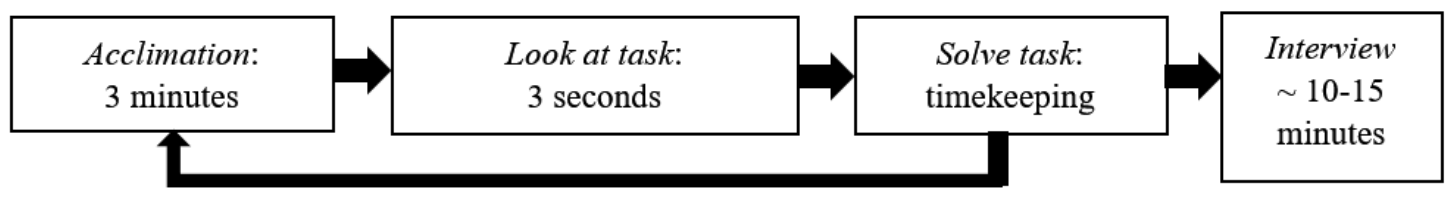

next input device + task

Figure 6. set-up for the evaluation process at University Hospital Tübingen 


\section{RESULTS}

\subsection{Requirements Engineering}

The requirements engineering process described in 2.1 lead to the requirements listed in table 1 and 2 :

Table 1. requirements for a $3 \mathrm{~d}$ input system for real time MRI guidance.

\begin{tabular}{|l|l|l|l|l|}
\hline Problem & Tasks & Circumstances & Goals/Expectations & Device \\
\hline Communication & $\begin{array}{l}\text { Control of } \\
\text { real-time MRI }\end{array}$ & Sterility & Better guiding of needle & Simple operated \\
& & MR-compatibility & More accurate + safer positioning & One-hand operation \\
& & $\begin{array}{l}\text { Lack of space } \\
\text { Sast connect }+\end{array}$ & Time savings & $\begin{array}{l}\text { Considering } \\
\text { tremble }\end{array}$ \\
\end{tabular}

Table 2. requirements for simulation software and for control options of input devices.

\begin{tabular}{|l|l|}
\hline $\begin{array}{l}\text { Requirements simulation } \\
\text { software }\end{array}$ & $\begin{array}{l}\text { Requirements for control options of } \\
\text { input devices }\end{array}$ \\
\hline 3D dataset & Switch: rotation/translation \\
\hline Needle in different positions & Switch between image planes \\
\hline $\begin{array}{l}\text { Image planes: coronar, axial, } \\
\text { sagittal }\end{array}$ & $\begin{array}{l}\text { Rotation in } 2 \text { spatial directions for each } \\
\text { image plane }\end{array}$ \\
\hline $\begin{array}{l}\text { Image artifacts of image } \\
\text { planes }\end{array}$ & $\begin{array}{l}\text { Translation in 1 spatial direction for } \\
\text { each image plane }\end{array}$ \\
\hline Frame rate: 2 images/sec & \\
\hline
\end{tabular}

\subsection{Evaluation}

The result of the pre-evaluation was that the Leap Motion Controller is not suitable at all and is not considered further for the simulation. The other input devices, Phantom Omni, Wii Remote, Space Navigator and Foot Switch were taken into account for the final evaluation.

The measured results of the evaluation with the interventionalists of University Hospital Tübingen are shown in Table 3. All measurements present a noticeable time reduction and furthermore reduction of costs. The best time of 34 seconds was achieved with WiiMote. The worst times were measured with SpaceNavigator and Foot Switch between 83 and 88 seconds. WiiMote reaches an average time of 50,5 seconds, SpaceNavigator of 70,5 seconds and Foot Switch of 65,25 seconds. It is noticeable that the third proband toke way longer than 1 minute to solve the exercise with WiiMote but proband 4 is the only one who solved the exercise with Foot Switch in less than a minute.

Based on the evaluation and the interviews the tendency goes to Wii Remote because the option doing it by hand seems more reasonable. The Wii Remote gives the user the possibility to lay it by side if it is not needed and has haptic feeling of the buttons while the Foot Switch is not so easy to handle if you cannot look at the input device on the ground.

Table 3. measured results of evaluation with interventionalists at University Hospital Tübingen.

\begin{tabular}{|l|l|l|l|l|l|}
\hline & Proband 1 & Proband 2 & Proband 3 & Proband 4 & Average \\
\hline WiiMote & $55 \mathrm{sec}$ & $34 \mathrm{sec}$ & $79 \mathrm{sec}$ & $34 \mathrm{sec}$ & $50,5 \mathrm{sec}$ \\
\hline SpaceNavigator & $88 \mathrm{sec}$ & $57 \mathrm{sec}$ & $54 \mathrm{sec}$ & $83 \mathrm{sec}$ & $70,5 \mathrm{sec}$ \\
\hline Foot switch & $84 \mathrm{sec}$ & $60 \mathrm{sec}$ & $73 \mathrm{sec}$ & $44 \mathrm{sec}$ & $65,25 \mathrm{sec}$ \\
\hline
\end{tabular}




\section{NEW OR BREAKTHROUGH WORK TO BE PRESENTED}

We built a simulation system for MRI based RFA which can be used to assess the quality of 3D controllers for real time MRI slice positioning tasks. We identified requirements for such input devices. The best input device WiiMote could be used for implementing it in real time MRI slice positioning during RFA.

\section{DISCUSSION}

The time reduction can be shown already with the measured results of the evaluation, but the needed time is still too much for regaining the needle. Now there is the question if the time savings could be improved if the interventionalists would complete some training sessions. On one hand in the daily business there is no time for long trainings. On the other hand, there is the possibility to save costs and time and furthermore to increase the safety. In the final interviews the interventionalists confirmed the easy learnability of the control even most of them did not use such a technology before. From this point of view the results of the evaluation can be seen positive and there should be time measurements of 10 to 20 seconds possible if the interventionalists are more familiar with the control.

Another point is the comparability with real interventions because of the simulated tasks and the trailing situation while the intervention. The tasks according to the interviews of final evaluation are comparable to real interventions. The difficult point is the difference between test and real environment. In the test environment the interventionalists are not holding a needle so they did not feel the strain. As well they were not leaning in an uncomfortable position at the MRI Scanner. Those circumstances could not be simulated in the evaluation so the final decision for a perfect fitting 3D-Controller can only be done when testing it under real conditions. This presented work can only make a recommendation for hand holding input devices like Wii Remote.

\section{CONCLUSIONS}

This paper presented a method for controlling a real-time MRI based on 3D-controller. The core idea was based on the time-consuming radiofrequency ablation with MRI as imaging technique. The issue was the impaired communication between medical-technical assistant and interventionalist due to loud gradient noises. Add to that in general the intervention would be more efficient if the interventionalist could do certain tasks by himself. Hence, 3D-controllers were selected and implemented in a simulation software. While pre-evaluation the Leap Motion Controller was not further considered as a suitable input device. The final evaluation toke place at the University Hospital Tübingen. The measured results showed that in general the control with 3D-controllers can reduce the needed time for radiofrequency ablation with MRI. It has shown that Wii Remote is the most appropriate 3D-controller for reducing the time consumption for radiofrequency ablation.

This work has not been submitted for publication or presentation elsewhere.

\section{REFERENCES}

[1] Handels H., [Medizinische Bildverarbeitung -Bildanalyse, Mustererkennung und Visualisierung für die computergestützte ärztliche Diagnostik und Therapie], Vieweg + Teubner, Wiesbaden, 10-20 (2009).

[2] Reimer, P., Parizel, P.M., Stichnoth, F.-A. [Klinische MR-Bildgebung - Eine praktische Anleitung], SpringerVerlag, Berlin Heidelberg, 20-35 (2000).

[3] Pinkernelle, J. G., Streitparth, F., Rump, J., Teichgräber, U., "Adaptation of a Wireless PC Mouse for Modification of GUI during Intervention in an Open Highfield MRI at 1.0T," Fortschr. Röntgenstr. 182(4), 348352 (2010).

[4] Mayer, H.O., [Interview und schriftliche Befragung - Grundlagen und Methoden empirischer Sozialforschung], Oldenbourg Verlag, München, 35-50 (2013). 